\title{
Quick Sequential (Sepsis Related) Organ Failure Assessment: A high performance rapid prognostication tool in patients having acute pyelonephritis with upper urinary tract calculi
}

\author{
Siddharth Pandey ${ }^{1}$, Satya Narayan Sankhwar ${ }^{1}$, Apul Goel ${ }^{1}$, Manoj Kumar ${ }^{1}$, Ajay Aggarwal ${ }^{1}$, Deepanshu Sharma ${ }^{1}$, \\ Samarth Agarwal ${ }^{1}$, Tushar Pandey ${ }^{2}$ \\ 'Department of Urology, King George's Medical University, Lucknow, ${ }^{2}$ Department of Pathology, Post Graduate Institute of Medical Education and Research, Chandigarh, \\ India
}

Purpose: To analyze the utility of quick Sequential Organ Failure Assessment (qSOFA) in patients with uro-sepsis due to acute pyelonephritis (APN) with upper urinary tract calculi, we conducted this study. The role of qSOFA as a tool for rapid prognostication in patients with sepsis is emerging. But there has been a great debate on its utility. Literature regarding utility of qSOFA in uro-sepsis is scarce.

Materials and Methods: Ours was a retrospective study including 162 consecutive patients who were admitted for APN with upper urinary tract calculi over a 3 and half years (total 42 months) period. We evaluated the accuracy of qSOFA in predicting inhospital mortality and intensive care unit (ICU) admissions and compared this with the predictive accuracy of systemic inflammatory response syndrome (SIRS). We used the Area Under Curve (AUC) of the Receiver Operator Characteristic curve to calculate it and also calculated the optimum cut off for qSOFA score.

Results: The overall mortality and ICU admission rates were $7.4 \%$ and $12.9 \%$, respectively. qSOFA had a higher predictive accuracy for in-hospital mortality (AUC, 0.981; 95\% confidence interval [CI], 0.962-1.000) and ICU admissions (AUC, 0.977; 95\% Cl, $0.955-$ 0.999) than SIRS. A qSOFA score of $\geq 2$ was an optimum cut off for predicting prognosis. In a multivariate model qSOFA $\geq 2$ was a highly significant predictor of in-hospital mortality and ICU admissions $(p<0.001)$.

Conclusions: qSOFA is a reliable and rapid bedside tool in patients with sepsis with accuracy more than SIRS in predicting inhospital mortality and ICU admissions.

Keywords: Hospital mortality; Pyelonephritis; Sepsis; Urinary tract infection; Urolithiasis

This is an Open Access article distributed under the terms of the Creative Commons Attribution Non-Commercial License (http://creativecommons.org/licenses/by-nc/4.0) which permits unrestricted non-commercial use, distribution, and reproduction in any medium, provided the original work is properly cited.

Received: 8 October, 2018 • Accepted: 6 January, 2019

Corresponding Author: Satya Narayan Sankhwar

Department of Urology, King George's Medical University, Chowk, Lucknow 226003, Uttar Pradesh, India

TEL: +91-9415007703, FAX: +91-522-2369841, E-mail: sankhwarsn.sn@gmail.com

ORCID: https://orcid.org/0000-0001-9875-5505 


\section{INTRODUCTION}

Diagnosis and management of sepsis has been an Achilles heel in any form of clinical practice. The aim in management of patients with sepsis is to recognize and treat them at an early stage so that its consequences can be prevented. To better define sepsis a consensus panel that included representatives from the American College of Chest Physicians and Society of Critical Care Medicine gave its recommendations in 1991 [1]. The definitions included that of systemic inflammatory response syndrome (SIRS), sepsis, severe sepsis and septic shock. These definitions were revised and expanded by another consensus panel in 2001 [2]. The most recent update to the definition of sepsis has been done by the Sepsis-3 task force in 2016 [3]. They defined sepsis as 'life threatening organ dysfunction caused by a dysregulated host response to infection'. They recommended the use of sequential (sepsis-related) organ failure assessment (Sequential Organ Failure Assessment, SOFA) as a measure of organ dysfunction. As the SOFA score is not universally accessible (especially for $\mathrm{PaO}_{2}$ which requires blood gas measurement), in addition they proposed the quick SOFA or quick Sequential Organ Failure Assessment (qSOFA) to rapidly assess the patient clinically for risk of deterioration due to sepsis, at bedside in wards or the emergency department outside the intensive care unit (ICU) [3].

Up to $30 \%$ of patients with sepsis have a urologic cause for sepsis (uro-sepsis) and the main reason for this is obstruction of the urinary tract [4-6]. Uro-sepsis (acute pyelonephritis, APN) due to obstructive upper urinary tract calculi is commonly encountered in urologic practice. In addition to antibiotics and other supportive measures it usually requires relief of obstruction with the help of a nephrostomy or a double $\mathrm{J}(\mathrm{JJ})$-stent [7,8]. Many times this disease requires management in an ICU and the mortality rate in literature has been reported to be as high as $10 \%$ $[7,9,10]$.

The qSOFA score has been an area of great debates, especially its utility in predicting in-hospital mortality. Some authors found qSOFA to have a better prognostic accuracy and others have reported SIRS criteria as described previously, to be more accurate than qSOFA in predicting in-hospital mortality [11-13]. We thus conducted this study to assess the utility of qSOFA in patients with uro-sepsis due to APN with upper urinary tract calculi.

\section{MATERIALS AND METHODS}

We retrospectively reviewed our prospectively main- tained database for patients who were admitted and treated for APN with upper urinary tract calculi between January 2015 and June 2018. The Institutional Review Board of King George's Medical University approved the study (approval number: 2981/Ethics/R-cell-18). Informed consent was taken from the patient. We could identify 162 such patients and thoroughly reviewed their medical records. In patients with multiple admissions for this disease we included the most severe episode. The patients with APN due to other causes including surgical interventions and those with bilateral APN were excluded from the study.

The diagnosis of APN was made on basis of clinical examination that included flank pain, tenderness and fever

Table 1. Characteristics of the study population

\begin{tabular}{lc}
\hline \multicolumn{1}{c}{ Characteristic } & Value \\
\hline Total patients & $162(100.0)$ \\
Age $(\mathrm{y})$ & $42(18-70)$ \\
Sex & \\
Male & $76(46.9)$ \\
Female & $86(53.1)$ \\
ECOG PS $>2$ & $14(8.6)$ \\
BMI $\left(\mathrm{kg} / \mathrm{m}^{2}\right)$ & $21(16-32)$ \\
BMI, $>25 \mathrm{~kg} / \mathrm{m}^{2}$ & $35(21.6)$ \\
Co-morbidities & \\
Diabetes mellitus & $60(37.0)$ \\
Hypertension & $27(16.7)$ \\
Immunosuppression & $16(9.9)$ \\
Stroke & $3(1.8)$ \\
Myocardial infarction & $4(2.5)$ \\
Malignancy & $8(4.9)$ \\
CCI $\geq 2$ & $71(43.8)$ \\
Laterality of APN & \\
Right & $74(45.7)$ \\
Left & $88(54.3)$ \\
Method of drainage & \\
PCN & $75(46.3)$ \\
Double J-stent & $65(40.1)$ \\
Conservative & $22(13.6)$ \\
TLC (/mm 3 ) & $12,200(1,400-27,700)$ \\
qSOFA & $0(0-3)$ \\
qSOFA 0-1 & $140(86.4)$ \\
qSOFA $\geq 2$ & $23(14.2)$ \\
SIRS & $3(0-4)$ \\
SIRS 0-1 & $12(7.4)$ \\
SIRS $\geq 2$ & $150(92.6)$ \\
\hline
\end{tabular}

Values are presented as number (\%) or median (range). ECOG PS, Eastern Cooperative Oncology Group performance status; $\mathrm{BMI}$, body mass index; $\mathrm{CCl}$, Charlson comorbidity index; APN, acute pyelonephritis; PCN, per-cutaneous nephrostomy; TLC, total leucocyte counts; qSOFA, quick Sequential Organ Failure Assessment; SIRS, systemic inflammatory response syndrome. 
along with raised total leucocyte counts (TLC), C-reactive protein, urine analysis and culture. The presence of upper urinary tract calculi was confirmed on ultrasonography (USG) and plain X-ray of kidney-ureter-bladder. In case of doubt a non-contrast computed tomography scan was done. We included patients both with obstructive and nonobstructive calculi. The patients were treated with antibiotics and other supportive measures. When decompression of the collecting system was required, either a double J-stent or USG guided per-cutaneous nephrostomy (PCN) was placed.

We collected the patient data that included their age, gender, Eastern Cooperative Oncology Group performance status (ECOG-PS), Charlson comorbidity index (CCI), body mass index (BMI), respiratory rate, body temperature, pulse rate, blood pressure, Glasgow Coma Scale (GCS), method of drainage, ICU admission and mortality. Mortality and ICU admissions were our primary and secondary outcomes respectively.

To calculate SIRS and qSOFA we used the worst physiological and laboratory data within the first 24 hours of admission. The criteria for SIRS included a temperature of $>38^{\circ} \mathrm{C}$ or $<36^{\circ} \mathrm{C}$, pulse rate $>90$ per minute, respiratory rate $>20$ breaths per minute and a TLC $>12,000$ or $<4,000 / \mathrm{mm}^{3}$ or $>10 \%$ immature forms (bands) [1]. For qSOFA the criteria included respiratory rate $>22$ breaths per minute, systolic blood pressure $<100 \mathrm{mmHg}$ and altered mental status with GCS $<15$ [3].

Statistical analysis was done using IBM SPSS Statistics ver. 21.0 software (IBM Co., Armonk, NY, USA). Continuous data was represented as median with range and categorical data with percentage. Chi-square test was used for categorical data and student t-test for continuous data. A p-value $<0.05$ was considered significant. For calculating the predictors of in-hospital mortality and ICU admissions, those clinical characteristics with more than 10 occurrences were analyzed in a univariate model. The significant variables were combined in a multivariate logistic regression analysis model. The accuracy of SIRS and qSOFA in predicting in-hospital mortality and ICU admissions was calculated using area under curve (AUC) of the receiver operating characteristic (ROC) curve. The optimal cut off and sensitivity and specificity for qSOFA and SIRS was

Table 2. Comparison of clinical characteristics in patients with and without mortality

\begin{tabular}{|c|c|c|c|}
\hline Characteristic & No mortality & Mortality & $\mathrm{p}$-value \\
\hline Total patients & $150(100.0)$ & $12(100.0)$ & - \\
\hline Age (y) & $42.5(18-70)$ & $49(40-68)$ & 0.005 \\
\hline Sex & & & 0.824 \\
\hline Male & $70(46.7)$ & $6(50.0)$ & \\
\hline Female & $80(53.3)$ & $6(50.0)$ & \\
\hline ECOG PS $>2$ & $6(4.0)$ & $6(50.0)$ & $<0.001$ \\
\hline BMI $\left(\mathrm{kg} / \mathrm{m}^{2}\right)$ & $21(16-32)$ & $21.25(17-30)$ & 0.829 \\
\hline $\mathrm{BMl},>25 \mathrm{~kg} / \mathrm{m}^{2}$ & $32(21.3)$ & $3(25.0)$ & 0.766 \\
\hline \multicolumn{4}{|l|}{ Co-morbidities } \\
\hline Diabetes mellitus & $50(33.3)$ & $10(83.3)$ & 0.001 \\
\hline Hypertension & $23(15.3)$ & $4(33.3)$ & 0.107 \\
\hline Immunosuppression & $12(8.0)$ & $4(33.3)$ & 0.005 \\
\hline $\mathrm{CCl}>2$ & $61(40.7)$ & $9(75.0)$ & 0.024 \\
\hline Method of drainage & & & 0.059 \\
\hline PCN & $67(44.7)$ & $8(66.7)$ & \\
\hline Double J-stent & $64(42.7)$ & $1(8.3)$ & \\
\hline Conservative & $19(12.7)$ & $3(25.0)$ & \\
\hline $\operatorname{TLC}\left(/ \mathrm{mm}^{3}\right)$ & $12,200(1,400-27,700)$ & $13,850(10,200-18,900)$ & 0.010 \\
\hline qSOFA & $0(0-3)$ & $2.5(2-3)$ & $<0.001$ \\
\hline qSOFA 0-1 & $139(92.7)$ & $0(0.0)$ & $<0.001$ \\
\hline$q S O F A \geq 2$ & $11(7.3)$ & $12(100.0)$ & \\
\hline SIRS & $2(0-4)$ & $4(3-4)$ & $<0.001$ \\
\hline SIRS 0-1 & $13(8.7)$ & $0(0.0)$ & 0.288 \\
\hline $\mathrm{SIRS} \geq 2$ & 137 (91.3) & $12(100.0)$ & \\
\hline
\end{tabular}

Values are presented as number (\%) or median (range).

ECOG PS, Eastern Cooperative Oncology Group performance status; BMI, body mass index; CCl, Charlson comorbidity index; PCN, per-cutaneous nephrostomy; TLC, total leucocyte counts; qSOFA, quick Sequential Organ Failure Assessment; SIRS, systemic inflammatory response syndrome. 
determined using co-ordinates of the ROC curve.

\section{RESULTS}

The median age of the study population was 42 years (range, 18-70 years) out of which 86 patients (53.1\%) were females. The median BMI was $21 \mathrm{~kg} / \mathrm{m}^{2}$ (range, 16 to $32 \mathrm{~kg} /$ $\mathrm{m}^{2}$ ) and 35 patients (21.6\%) had a BMI of more than $25 \mathrm{~kg} /$ $\mathrm{m}^{2}$. There were 14 patients (8.6\%) with an ECOG-PS of greater than 2 . The most common associated comorbidity was diabetes mellitus (DM), which was present in 60 patients (37.0\%) followed by hypertension, which was present in 27 patients (16.7\%). Seventy-one patients (43.8\%) had a CCI of greater than 2. Right-sided APN was present in 74 patients (45.7\%) and 88 patients (54.3\%) had left sided disease. The most common method of drainage was PCN placement, which was done in 75 (46.3\% patients). A double J-stent was placed in 65 patients (40.1\%) and rest, were managed conservatively. The characteristics are summarized in Table 1.
A qSOFA score of $\geq 2$ was present in $14.2 \%$ patients whereas a SIRS score of $\geq 2$ was present in $92.6 \%$ patients. Eighty two percent patients with qSOFA $\geq 2$ and $84.7 \%$ patients with SIRS $\geq 2$ underwent drainage of the pelvi-calyceal system. A greater proportion of patients (52.0\%) with qSOFA

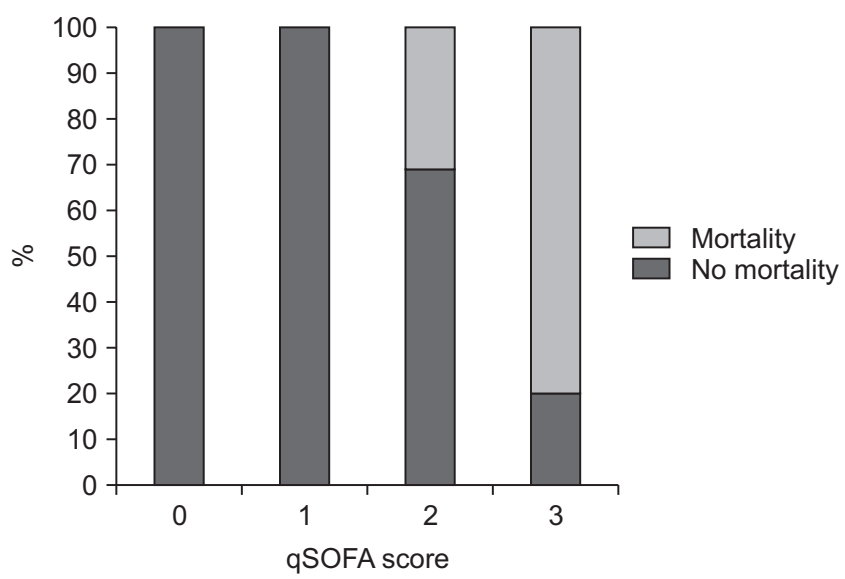

Fig. 1. Distribution of patient mortality according to quick Sequential Organ Failure Assessment (qSOFA) score.

Table 3. Comparison of clinical characteristics in patients with and without ICU admissions

\begin{tabular}{|c|c|c|c|}
\hline & No ICU admission & ICU admission & p-value \\
\hline Total patients & $141(100.0)$ & $21(100.0)$ & \\
\hline Age $(y)$ & $40(18-70)$ & $49(21-65)$ & 0.018 \\
\hline Sex & & & 0.945 \\
\hline Male & $66(46.8)$ & $10(47.6)$ & \\
\hline Female & $75(53.2)$ & $11(52.4)$ & \\
\hline ECOG PS $>2$ & $3(2.1)$ & $11(52.4)$ & $<0.001$ \\
\hline BMI $\left(\mathrm{kg} / \mathrm{m}^{2}\right)$ & $21(16-32)$ & $20(17.5-30)$ & 0.698 \\
\hline $\mathrm{BMl},>25 \mathrm{~kg} / \mathrm{m}^{2}$ & $30(21.3)$ & $5(23.8)$ & 0.792 \\
\hline \multicolumn{4}{|l|}{ Co-morbidities } \\
\hline Diabetes mellitus & $46(32.6)$ & $14(66.7)$ & 0.003 \\
\hline Hypertension & $20(14.2)$ & $7(33.3)$ & 0.028 \\
\hline Immunosuppression & $8(5.7)$ & $8(38.1)$ & $<0.001$ \\
\hline $\mathrm{CCl}>2$ & $52(36.9)$ & $19(90.5)$ & $<0.001$ \\
\hline Method of drainage & & & 0.841 \\
\hline $\mathrm{PCN}$ & $65(46.1)$ & $10(47.6)$ & \\
\hline Double J-stent & $56(39.7)$ & $9(42.9)$ & \\
\hline Conservative & $20(14.2)$ & $2(9.5)$ & \\
\hline $\operatorname{TLC}\left(/ \mathrm{mm}^{3}\right)$ & $12,200(1,400-27,700)$ & $11,200(4,200-20,200)$ & 0.627 \\
\hline qSOFA & $0(0-2)$ & $2(1-3)$ & $<0.001$ \\
\hline qSOFA 0-1 & $137(97.2)$ & $2(9.5)$ & $<0.001$ \\
\hline$q S O F A \geq 2$ & $4(2.8)$ & $19(90.5)$ & \\
\hline SIRS & $2(0-4)$ & $3(3-4)$ & $<0.001$ \\
\hline SIRS 0-1 & $13(9.2)$ & $0(0.0)$ & 0.147 \\
\hline SIRS $\geq 2$ & $128(90.8)$ & $21(100.0)$ & \\
\hline
\end{tabular}

Values are presented as number (\%) or median (range).

ICU, intensive care unit; ECOG PS, Eastern Cooperative Oncology Group performance status; BMI, body mass index; $\mathrm{CCl}$, Charlson comorbidity index; PCN, per-cutaneous nephrostomy; TLC, total leucocyte counts; qSOFA, quick Sequential Organ Failure Assessment; SIRS, systemic inflammatory response syndrome. 
of $\geq 2$ underwent drainage by $\mathrm{PCN}$ placement as compared to patients with $\operatorname{SIRS} \geq 2(44.7 \%)$. The overall mortality rate and ICU admission rates were $7.4 \%$ and $12.9 \%$, respectively. The association of various clinical characteristics with inhospital mortality and ICU admissions is given in Tables 2 and 3. Patients with a higher qSOFA had a highly significant $(p<0.001)$ rate of in-hospital mortality and ICU admissions. The distribution of in-hospital mortality and ICU admissions according to the qSOFA score is give in Figs. 1 and 2.

The predictive accuracy of qSOFA (AUC, 0.981; 95\% confidence interval [CI], 0.962-1.000) for in-hospital mortality was higher than that of SIRS (AUC, 0.924; 95\% CI, 0.860-0.989). Similarly for ICU admissions qSOFA

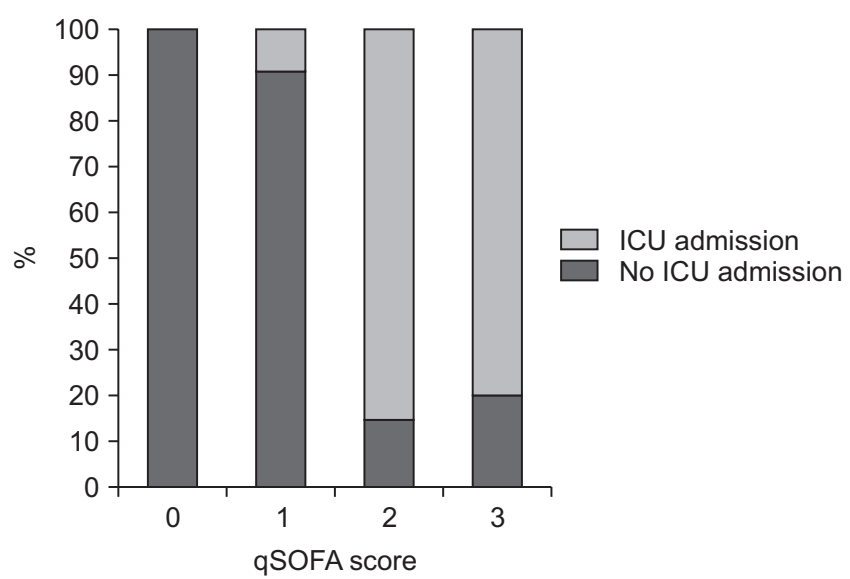

Fig. 2. Distribution of intensive care unit (ICU) admission according to quick Sequential Organ Failure Assessment (qSOFA) score.
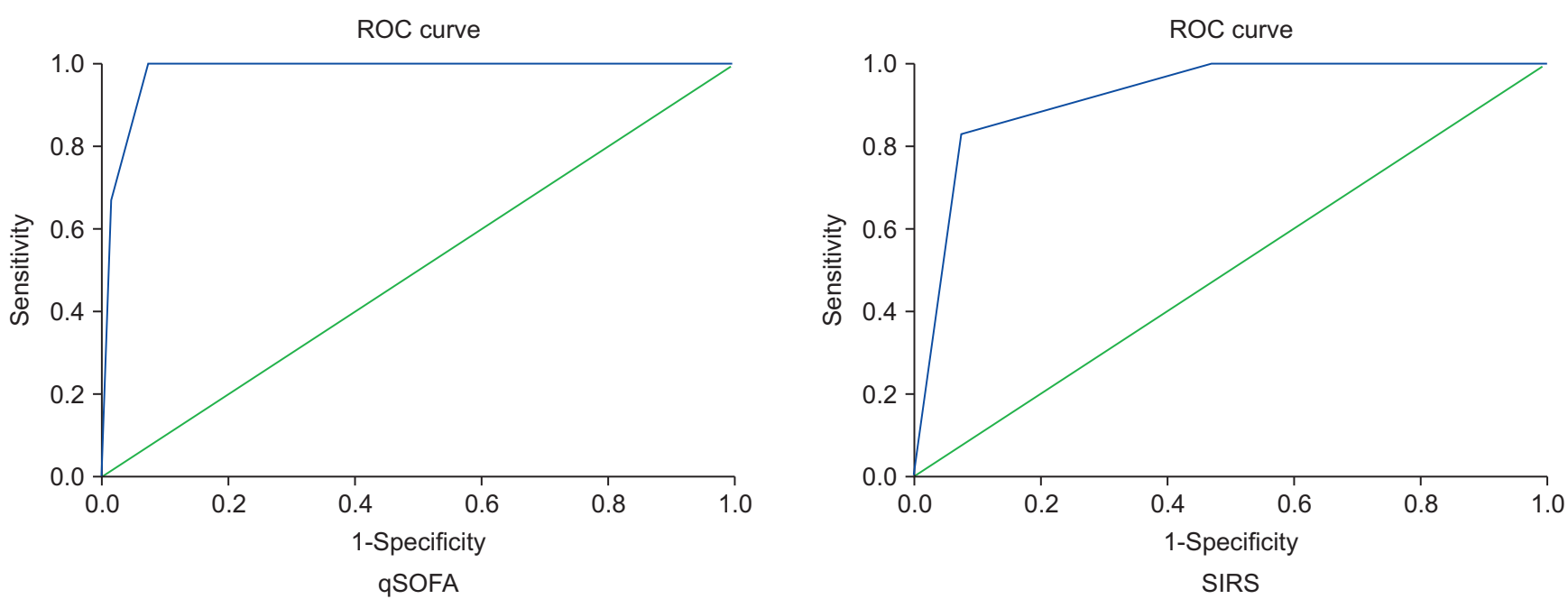

In-hospital mortality
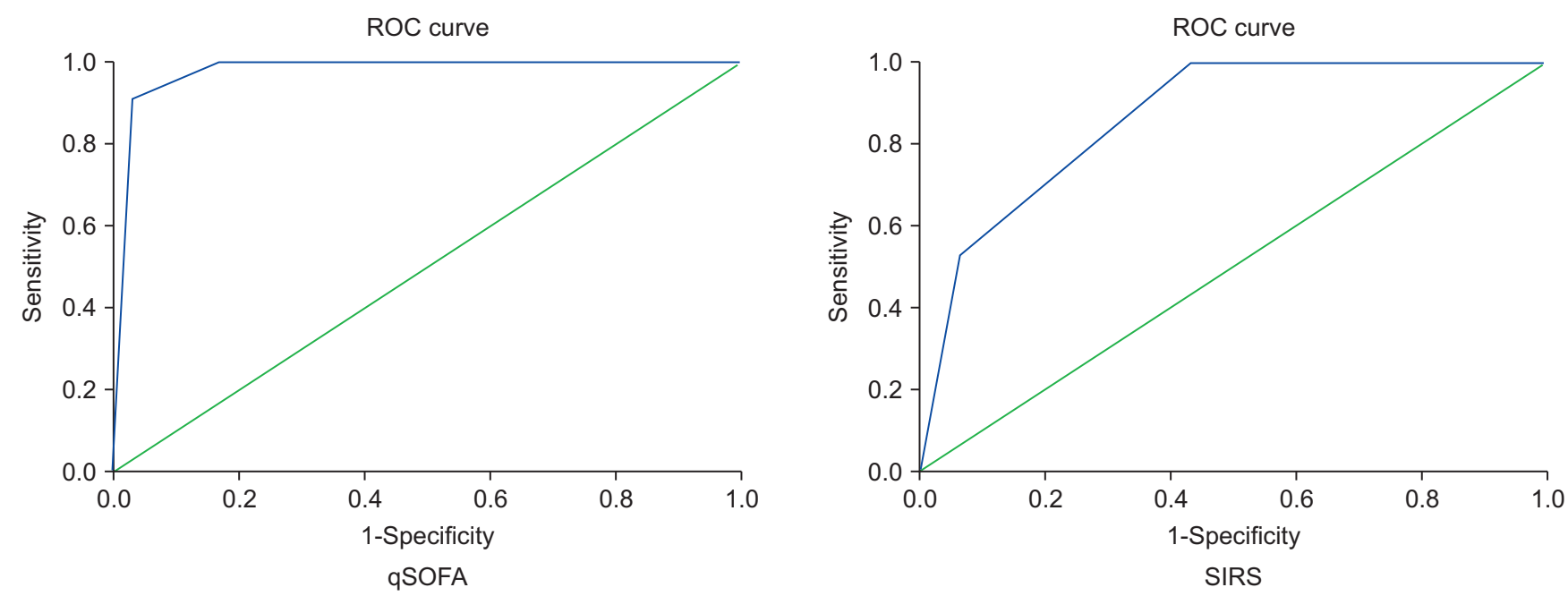

ICU admissions

Fig. 3. qSOFA (AUC, 0.981; $95 \% \mathrm{Cl}, 0.962-1.000$ ) and SIRS (AUC, 0.924; $95 \% \mathrm{Cl}, 0.860-0.989$ ) ROC curves for in-hospital mortality on top and qSOFA (AUC, 0.977; 95\% Cl, 0.955-0.999) and SIRS (AUC, 0.860; 95\% Cl, 0.792-0.928) ROC curves for ICU admissions on the bottom. qSOFA, quick Sequential Organ Failure Assessment; AUC, area under curve; Cl, confidence interval; SIRS, systemic inflammatory response syndrome; ROC, receiver operating characteristic; ICU, intensive care unit. 
Table 4. Multivariate logistic regression analysis of predictors of in-hospital mortality and ICU admissions

\begin{tabular}{cccc}
\hline Variable & OR & $95 \% \mathrm{Cl}$ & p-value \\
\hline $\begin{array}{c}\text { Mortality } \\
\text { qSOFA } \geq 2\end{array}$ & 40.03 & $1.734-924.225$ & 0.021 \\
$\begin{array}{c}\text { ICU Admission } \\
\text { qSOFA } \geq 2\end{array}$ & 163.64 & $11.719-2,285.140$ & 0.003 \\
\hline
\end{tabular}

ICU, intensive care unit; OR, odds ratio; $\mathrm{Cl}$, confidence interval; qSOFA, quick Sequential Organ Failure Assessment.

(AUC, 0.977; 95\% CI, 0.955-0.999) had a higher predictive accuracy than SIRS (AUC, 0.860; 95\% CI, 0.792-0.928). A qSOFA score of $\geq 2$ had a sensitivity and specificity of $100 \%$ and $93 \%$ respectively for in-hospital mortality and $90 \%$ and $97 \%$ for ICU admissions, whereas the same for SIRS $\geq 2$ was $100 \%$ and $66 \%$ for in-hospital mortality and $100 \%$ and $63 \%$ for ICU admissions. The ROC curves of SIRS and qSOFA are depicted in Fig. 3. The predictive value of qSOFA $\geq 2$ in a multivariate logistic regression model is given in Table 4.

\section{DISCUSSION}

Although the qSOFA score as recommended by the Sepsis-3 task is an easy bedside tool for assessing the prognosis of patients with sepsis, its prognostic accuracy has remained an area of debate. The SOFA score is a better measure of outcome in patients with sepsis than qSOFA and SIRS, but usually it is more relevant in an ICU setting as it requires a number of laboratory tests and clinical parameters. This led to the recommendation of using the qSOFA score, which is rapid and more relevant in the ward setting especially when there are limited resources [3,13]. The qSOFA score has found to have a low sensitivity for identifying patients with sepsis, which is a major limitation to its utility [14,15]. One study also found it to have a low sensitivity of predicting mortality due to sepsis [15]. When compared to the SIRS score, the qSOFA score has a lower sensitivity but higher specificity for predicting mortality in patients with sepsis [16,17] The general consensus is that SIRS is too sensitive to predict outcomes in patients with sepsis and thus the requirement of better scores.

A fair argument may be that severe sepsis is more specific in predicting ICU admissions and mortality in patients with sepsis. This has also been proven in previous studies [18]. But evaluating all elements to diagnose patients with severe sepsis may not be possible in non-ICU settings, especially in rural areas or centers with constraint of resources (which is also the case with SOFA score as mentioned vide supra). The assessments of mental status and blood pressure in qSOFA score are also parameters used to assess severe sepsis [1]. Being a retrospective study we could not compare qSOFA and severe sepsis, but it would be reasonable to say that due to its simplicity qSOFA would be a better predictor of ICU admissions and mortality than both SIRS and severe sepsis.

Treating patients with uro-sepsis is an integral part of urologic practice and it is pertinent that a urologist is aware and up to-date with the various tools in assessing patients with sepsis. Our study found qSOFA score to be a very useful tool in predicting the outcome of patients with urosepsis because of APN with upper urinary tract calculi. The specificity and predictive accuracy of qSOFA score were found to be higher than the SIRS score. A qSOFA score of $\geq 2$ was found to be an optimum cut off for predicting the inhospital mortality and ICU admissions. This would improve the patient management as those with higher qSOFA can be transferred to the ICUs earlier with aggressive management improving the outcome of these patients.

qSOFA is calculated purely on clinical parameters and no laboratory tests are required in its calculation unlike SIRS. According to one study the altered mental state used to assess qSOFA had a higher odds ratio to predict inhospital mortality and this explains the higher predictive performance of qSOFA over SIRS [6]. Literature on the utility of these new scores in patients with urologic cause of sepsis is lacking and our study could be a valuable addition to that.

Our study has certain important limitations. Firstly, it is a retrospective study with a limited sample size carried out in a single institute. A larger, multi-institutional prospective study would better validate the utility of qSOFA as a rapid bedside assessment tool in this cohort of patients. Secondly, we could not analyze the SOFA score as it requires a host of clinical and laboratory parameters which were not available for all patients. Thirdly, our institute is a state run tertiary care center in Northern India, which mainly caters to patients from rural areas with lower socio-economic status and thus our data is more pertinent to that subset of patient population.

\section{CONCLUSIONS}

The role of qSOFA as proposed by the Sepsis-3 task force, as a rapid bedside assessment tool in patients with sepsis replacing the SIRS score has been a matter of debate in recent literature. Our study finds qSOFA to be better tool than SIRS having a high predictive accuracy in predicting 
in-hospital mortality and ICU admission in patients with uro-sepsis because of APN due to upper urinary tract calculi.

\section{CONFLICTS OF INTEREST}

The authors have nothing to disclose.

\section{REFERENCES}

1. Bone RC, Balk RA, Cerra FB, Dellinger RP, Fein AM, Knaus WA, et al. Definitions for sepsis and organ failure and guidelines for the use of innovative therapies in sepsis. The ACCP/ SCCM Consensus Conference Committee. American College of Chest Physicians/Society of Critical Care Medicine. Chest 1992;101:1644-55.

2. Levy MM, Fink MP, Marshall JC, Abraham E, Angus D, Cook D, et al. 2001 SCCM/ESICM/ACCP/ATS/SIS International Sepsis Definitions Conference. Crit Care Med 2003;31:1250-6.

3. Singer M, Deutschman CS, Seymour CW, Shankar-Hari M, Annane D, Bauer M, et al. The Third International Consensus Definitions for Sepsis and Septic Shock (Sepsis-3). JAMA 2016;315:801-10.

4. Naber KG. Urogenital infections: the pivotal role of the urologist. Eur Urol 2006;50:657-9.

5. Brun-Buisson C. The epidemiology of the systemic inflammatory response. Intensive Care Med 2000;26 Suppl 1:S64-74.

6. Fukushima H, Kobayashi M, Kawano K, Morimoto S. Performance of quick sequential (sepsis related) and sequential (sepsis related) organ failure assessment to predict mortality in patients with acute pyelonephritis associated with upper urinary tract calculi. J Urol 2018;199:1526-33.

7. Yoshimura K, Utsunomiya N, Ichioka K, Ueda N, Matsui Y, Terai A. Emergency drainage for urosepsis associated with upper urinary tract calculi. J Urol 2005;173:458-62.

8. Pearle MS, Pierce HL, Miller GL, Summa JA, Mutz JM, Petty $\mathrm{BA}$, et al. Optimal method of urgent decompression of the collecting system for obstruction and infection due to ureteral calculi. J Urol 1998;160:1260-4.

9. Hamasuna R, Takahashi S, Nagae H, Kubo T, Yamamoto S, Arakawa S, et al. Obstructive pyelonephritis as a result of urolithiasis in Japan: diagnosis, treatment and prognosis. Int J Urol 2015;22:294-300.
10. Yamamoto Y, Fujita K, Nakazawa S, Hayashi T, Tanigawa G, Imamura $\mathrm{R}$, et al. Clinical characteristics and risk factors for septic shock in patients receiving emergency drainage for acute pyelonephritis with upper urinary tract calculi. BMC Urol 2012;12:4.

11. Freund $Y$, Lemachatti N, Krastinova E, Van Laer M, Claessens YE, Avondo A, et al. Prognostic accuracy of Sepsis-3 criteria for in-hospital mortality among patients with suspected infection presenting to the emergency department. JAMA 2017;317:301-8.

12. Raith EP, Udy AA, Bailey M, McGloughlin S, MacIsaac C, Bellomo R, et al. Prognostic accuracy of the SOFA score, SIRS criteria, and qSOFA score for in-hospital mortality among adults with suspected infection admitted to the intensive care unit. JAMA 2017;317:290-300.

13. Sartelli M, Kluger Y, Ansaloni L, Hardcastle TC, Rello J, Watkins RR, et al. Raising concerns about the Sepsis-3 definitions. World J Emerg Surg 2018;13:6.

14. Tusgul S, Carron PN, Yersin B, Calandra T, Dami F. Low sensitivity of qSOFA, SIRS criteria and sepsis definition to identify infected patients at risk of complication in the prehospital setting and at the emergency department triage. Scand J Trauma Resusc Emerg Med 2017;25:108.

15. Askim Å, Moser F, Gustad LT, Stene H, Gundersen M, Åsvold $\mathrm{BO}$, et al. Poor performance of quick-SOFA (qSOFA) score in predicting severe sepsis and mortality-a prospective study of patients admitted with infection to the emergency department. Scand J Trauma Resusc Emerg Med 2017;25:56.

16. Henning DJ, Puskarich MA, Self WH, Howell MD, Donnino MW, Yealy DM, et al. An emergency department validation of the SEP-3 sepsis and septic shock definitions and comparison with 1992 consensus definitions. Ann Emerg Med 2017;70:54452.e5.

17. Giamarellos-Bourboulis EJ, Tsaganos T, Tsangaris I, Lada M, Routsi C, Sinapidis D, et al. Validation of the new Sepsis-3 definitions: proposal for improvement in early risk identification. Clin Microbiol Infect 2017;23:104-9.

18. Sartelli M, Catena F, Ansaloni L, Coccolini F, Corbella D, Moore EE, et al. Complicated intra-abdominal infections worldwide: the definitive data of the CIAOW Study. World J Emerg Surg 2014;9:37. 